\title{
Find Your Center: Using Engineering and Biomechanics to Investigate Cen- ter of Mass (P12 Resource Exchange)
}

\section{Dr. Amy Trauth-Nare, University of Delaware}

Amy Trauth-Nare, Ph.D., is the Associate Director of Science Education at the University of Delaware's Professional Development Center for Educators. In her role, Amy works collaboratively with K-12 science and engineering teachers to develop and implement standards-based curricula and assessments. She also provides mentoring and coaching and co-teaching support to K-12 teachers across the entire trajectory of the profession. Her research focuses on teacher education, classroom assessment, and P-16 environmental and engineering education.

\section{Prof. Jenni Buckley, University of Delaware}

Dr. Buckley is an Assistant Professor of Mechanical Engineering at University of Delaware. She received her Bachelor's of Engineering (2001) in Mechanical Engineering from the University of Delaware, and her MS (2004) and PhD (2006) in Mechanical Engineering from the University of California, Berkeley, where she worked on computational and experimental methods in spinal biomechanics. Since 2006, her research efforts have focused on the development and mechanical evaluation of medical and rehabilitation devices, particularly orthopaedic, neurosurgical, and pediatric devices. She teaches courses in design, biomechanics, and mechanics at University of Delaware and is heavily involved in K12 engineering education efforts at the local, state, and national levels. 


\section{FIND YOUR CENTER: USING STEM PRINCIPLES TO EXPLORE CENTER-OF-MASS}

Amy Trauth-Nare ${ }^{1}$, Emily Pavilonis²,4; Julia Paganucci³; Gemma Ciabattoni³; Jenni M. Buckley ${ }^{1,3,4}$

${ }^{1}$ Professional Development Center for Educators, University of Delaware, Newark, DE 19716

${ }^{2}$ Department of Biomedical Engineering, University of Delaware, Newark, DE 19716 ${ }^{3}$ Department of Mechanical Engineering, University of Delaware, Newark, DE 19716 ${ }^{4}$ The Perry Initiative, San Francisco, CA 94117, USA 


\begin{abstract}
In this article, we describe how to teach center of mass principles in the context of athletic performance. Our goal was to provide relevant lessons on biomechanics, a subfield of mechanics in engineering and physics that deal with forces and motion related to the human body. Biomechanics represents a unique learning opportunity to teach core mechanics concepts through the lens of anatomy and medicine, which is more relatable to students than classic examples from automotive and aerospace engineering. In this article, we include a $5 \mathrm{E}$ lesson plan for engaging high school students in core ideas, crosscutting concepts and science and engineering practices related to force and motion in the Next Generation Science Standards. Students apply three methods for estimating center of mass and apply their understanding of center of mass to make recommendations to a diver for safe diving distances.
\end{abstract}




\section{Introduction}

Mechanics is a branch of engineering and physics that deal with forces and motion, and its fundamental principles apply to all solid objects, whether it is a bouncing ball, a bicycle, or the human body. The field of biomechanics specifically applies mechanics concepts to the human body, and understanding of biomechanics is important for many careers such as biomedical engineering, physical therapy and athletic training, orthopaedic surgery, and prosthetics. Biomechanics also represents a unique learning opportunity to teach core mechanics concepts through the lens of anatomy and medicine, which is more relatable to students, particularly underrepresented groups in STEM, than classic examples from automotive and aerospace engineering.

In this NGSS-aligned lesson, students first learn about center-of-mass (COM), a critical location on an object that affects its balance and motion (Table 1). Students engage with everyday objects, such as a pencil and a boomerang, to gain an intuitive understanding of unique features of COM and how to approximate and measure COM location. Students apply this knowledge to a real world example of biomechanics, specifically, coaching a beginning diver to avoid contact with the diving board during flip dives. Using a simple, doit-yourself balance board, students conduct an investigation to determine COM location on themselves and their classmates in various diving positions, analyze and interpret data, and use computational reasoning to recommend a safe jumping distance for the diver. Students think critically, develop and test hypotheses about what factors affect the diver's risk of contacting the board, such as type of dive and height of diver. Supplemental materials and student handouts are available for this lesson on NSTA Connections. 
Table 1. Connections to the Next Generation Science Standards, Common Core Mathematics and English Language Arts.

\section{Standard \\ PS2.A: Forces and Motion}

\section{Performance Expectation}

The activities in this article are just one step towards reaching this performance expectation: HS-PS2-1. Analyze data to support the claim that Newton's second law of motion describes the mathematical relationship among net force on the macroscopic object, its mass and its acceleration.

\begin{tabular}{l|l|l}
\hline Dimension & Standard & Student Activity \\
\hline $\begin{array}{l}\text { Disciplinary } \\
\text { Sorea }\end{array}$ & PS2.A Forces and Motion \\
$\begin{array}{l}\text { Engineering } \\
\text { Practices }\end{array}$ & $\begin{array}{l}\text { Planning and carrying out } \\
\text { investigations } \\
\text { Analyzing and interpreting } \\
\text { data } \\
\text { Using mathematics and } \\
\text { computational thinking } \\
\text { Developing and using models } \\
\text { Constructing explanations } \\
\text { and designing solutions }\end{array}$ & $\begin{array}{l}\text { Students find their center of mass, and } \\
\text { then analyze and interpret data from their } \\
\text { classmates and a larger data set. }\end{array}$ \\
$\begin{array}{l}\text { Crosscutting } \\
\text { Concept }\end{array}$ & $\begin{array}{l}\text { Ptudents use the derived moment } \\
\text { equation to calculate individuals' center of }\end{array}$ \\
\hline Patterns & $\begin{array}{l}\text { Students develop written explanations for } \\
\text { trends in class data, discerning differences } \\
\text { based on demographic variables. }\end{array}$ \\
\hline
\end{tabular}

Connections to the Common Core State Standards (NGAC and CCSSO, 2010)

English Language Arts

WHST.11-12.2 Write informative/explanatory texts, including the narration of historical events, scientific procedures/experiments, or technical processes.

WHST.11-12.7 Conduct short as well as more sustained research projects to answer a question (including a self-generated question) or solve a problem; narrow or broaden the inquiry when appropriate; synthesize multiple sources on the subject, demonstrating understanding of the subject under investigation.

WHST.11-12.9 Draw evidence from informational texts to support analysis and research. Mathematics

MP.2 Reason abstractly and quantitatively

MP.4 Model with mathematics

HSN.Q.A.2. Define appropriate quantities for the purpose of descriptive modeling. HSA.SSE.A.1 Interpret expressions that represent a quantity in terms of its context. HSA.CED.A.4 Rearrange formulas to highlight a quantity of interest, using the same reasoning as in solving equations.

HSF-IF.C.7 Graph functions expressed symbolically and show key features of the graph, by hand in simple cases and using technology for more complicated cases.

HSS-IS.A.1 Represent data with plots on the real number line (dot plots, histograms, etc.) 


\section{Background}

Center-of-mass (COM) is the balance point of an object. Every object has a center of mass, and there are several interesting features associated with this special point in space. First, the entire mass of an object can be modeled as acting through its COM. This means if you suspend an object at its COM, it will balance perfectly and not spin. In this way, COM location is important to the stability of any object. The second feature of COM is that it affects the motion of an object when it is thrown in the air or pushed and then not acted upon by any other outside forces. The object will then spin about its COM, and the motion of the object can be modeled as translation combined with rotation about the COM. COM does not necessarily need to be located directly on an object; it may actually be outside of the object. A good example of this is a flying disc with a hollow center. The COM of this object will be in the middle of the disc, where there is no material. This will be the natural balance point and point of rotation of the object.

There are three ways to locate the COM for any object. The first is a simple balance test, which we have all performed at some time or another. If you try to balance a small object, like a pencil, on your finger, you will need to move the object around until you find the balance point. This point corresponds to the COM of that object and, in engineering, COM location is generally represented with a yellow and black checked target, like the targets representing the COM location of various body segments of a crash test dummy. A second way to approximate COM location is to apply your understanding of the object's inherent geometry, symmetry, and mass distribution. A good example would be a boomerang (Figure 1). The two arms of the boomerang are symmetrical, so the COM would be located somewhere along the line of symmetry. 


\section{Figure 1.}

Estimating the center-of-mass (COM) location of a boomerang, by identifying line of symmetry; recognizing underlying geometry, which is a triangle, and finding the geometric center of the triangle; and estimating COM location to be slightly above geometric center of the triangle because there is more mass above than below the geometric center (shown in red).

Step \#1: Symmetry

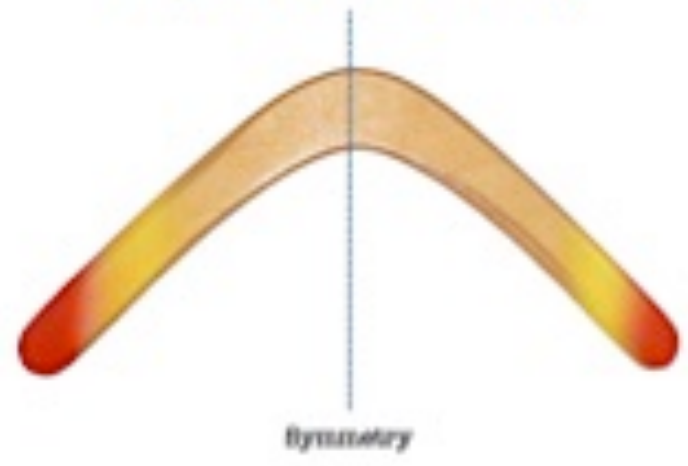

Step \#2: Geometry

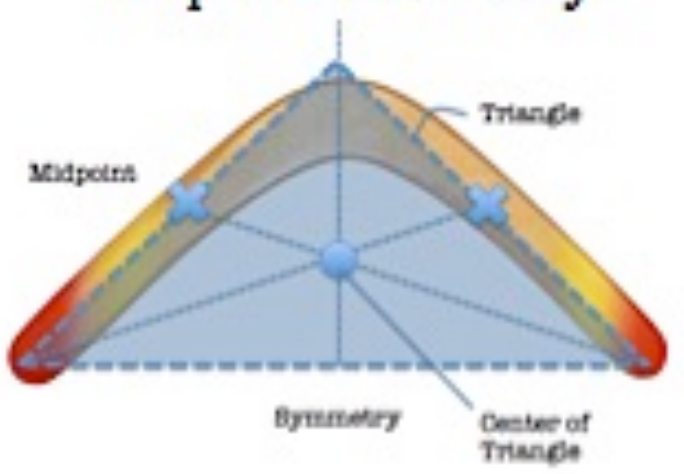

\section{Step \#3: Mass Distribution}

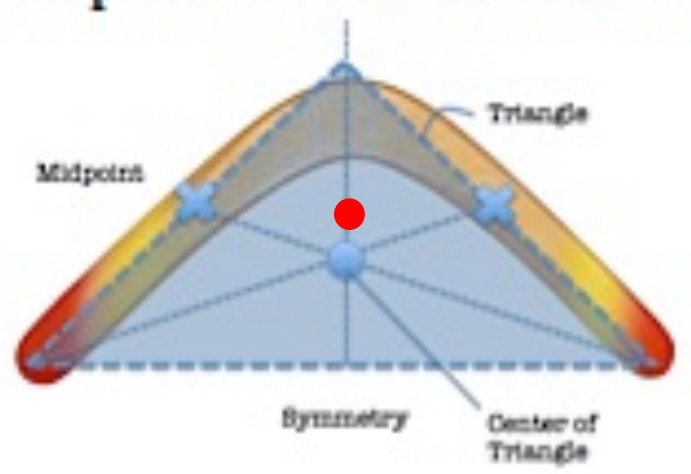


The overall geometry of the boomerang is similar to an isosceles triangle, and we can find the geometric center of a triangle by connecting the midpoint of each side to the opposite vertex. Along the line of symmetry, there is more mass of the boomerang above the geometric center of the triangle than below it; therefore, the COM will be located slightly above the geometric centroid of the triangle.

The third and final way to measure COM location is to use a simple balance board (Figure 2). A balance board consists of two scales, scale \#1 measuring weight $\mathrm{W}_{1}$ and scale \#2 measuring $\mathrm{W}_{2}$ separated by a distance, $\mathrm{L}$, upon which you place a rigid board. The scales are zeroed to subtract the weight of the board. The object for which you want to determine COM location is placed upon the board, and the differences in the readings on the scales determines COM location according to the Balance Board Equation, shown below.

Figure 2. Balance board setup for finding center of mass of an object. The COM location of any object can be calculated from $\mathrm{W}_{1}, \mathrm{~W}_{2}$, and $\mathrm{L}$, as the distance $\mathrm{d}$ (distance from scale \#1 to the COM), where: $d=\frac{W_{2}}{w_{1}+W_{2}} L$

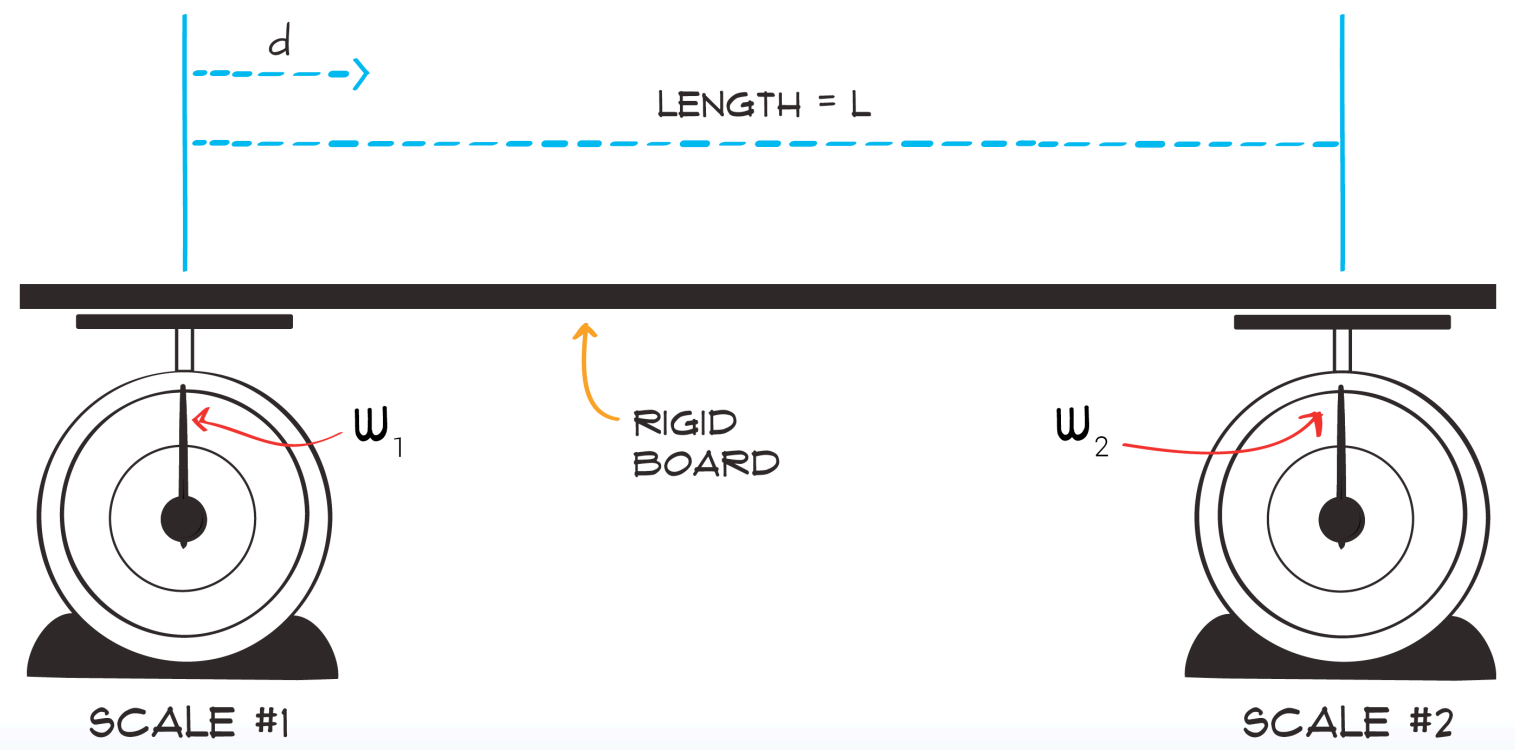


There are two methods for deriving the Balance Board Equation, and full derivations using both methods are presented in supplemental online materials (see Supplemental File). The first method applies basic algebra and pattern recognition skills, while the second, more advanced method uses algebra and basic mechanics concepts from physics. In the first method, a thought experiment can be conducted in which an unknown weight, $\mathrm{W}$, is placed on the balance board at various locations and the readings on the scales are estimated. For example, placing the weight, $\mathrm{W}$, at the mid-point of the board ( $\mathrm{d}=$ $1 / 2 \mathrm{~L}$ ) would result in $\mathrm{W}_{1}=\mathrm{W}_{2}=1 / 2 \mathrm{~W}$. The Balance Board Equation may be derived by recognizing multiple patterns across the thought experiment. The second method for deriving the Balance Board Equation involves conducting a static analysis of the balance board itself. There are three forces acting on the board, namely, $\mathrm{W}_{1}, \mathrm{~W}_{2}$, and $\mathrm{W}$, where $\mathrm{W}$ is the total weight of the object and $\mathrm{W}=\mathrm{W}_{1}+\mathrm{W}_{2}$ through force equilibrium. The Balance Board Equation can be determined from the moment equilibrium as:

$$
\sum h_{b}=0=-d w_{1}+(L-d) w_{2} \rightarrow d=\frac{w_{2}}{w_{1}+W_{2}} L
$$

\section{Engage (60 min)}

In the first part of this lesson, students gain a fundamental understanding of the special features of the center-of-mass (COM) of an object and experience the three different ways of measuring COM location, namely: (1) balance test with everyday objects; (2) using geometry, symmetry, and mass distribution; and (3) balance board experiment. COM definition and features are first explained in the context of an everyday object, a sharpened pencil. Students estimate the pencil's COM based on these variables and then conduct a 
balance test by balancing the pencil on the tip of their finger and mark the COM location with colored tape on the sharpened pencil (Figure 3).

Figure 3. Guiding questions on pencil COM for students during Engage phase activity.

1) Sketch your pencil. Note on your sketch the important geometric features: total length, midpoint of the pencil, and length of pencil point. Predict the location of the center of mass of the pencil based on what you know. Write your prediction in the table below.

2) Perform a balance test to check your prediction. To do this, extend your index finger and balance the pencil on the tip of your finger. Once it's balanced, use a piece of colored tape to mark the point on the pencil where it is balanced on your finger. Record the actual center of mass (i.e., tape mark) in your data table.

\begin{tabular}{|c|c|}
\hline & $\begin{array}{c}\text { Distance from pencil } \\
\text { point }(\mathrm{cm})\end{array}$ \\
\hline mid-point & \\
\hline estimated COM & \\
\hline actual COM & \\
\hline
\end{tabular}

3) Flick the pencil across the pencil across a large horizontal surface, e.g., lab table or floor. Track the motion of the COM. Perform this test several times. Record your observations below with a sketch of the path of COM and write an explanation below your sketch.

Because the pencil is sharpened (less mass) and has an eraser (more mass), the COM is located near the geometric mid-point of the pencil, slightly closer to the eraser. Then, students flick the pencil across a smooth, horizontal surface to observe that the COM follows a linear path with the ends of the pencil rotating around it (Figure 4). This quick exercise demonstrates the importance of COM location in balance and motion of an object. 
Students practice estimating COM location by examining the geometry, symmetry, and mass distribution of three everyday objects. The boomerang is first presented as an example of an object where all three features are incorporated into COM estimation.

Figure 4. COM trajectory of pencil during flick test.

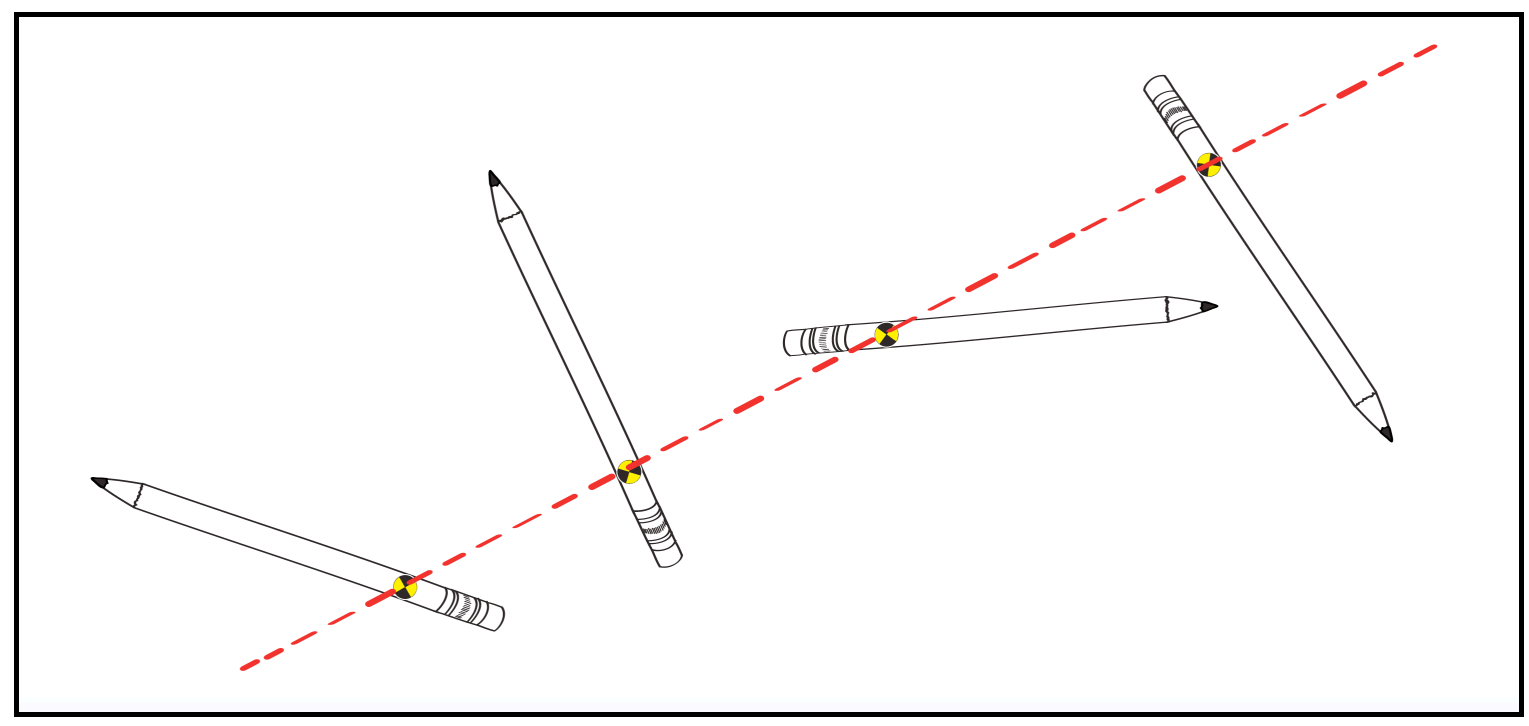

Students should first mark the line of symmetry and then find the geometric center of the boomerang by tracing a triangle and drawing lines from the midpoints of the triangle legs. Finally, students should consider where the majority of the boomerang's mass is located in relation to its geometric center. The boomerang illustrates that COM can be located anywhere in space, not necessarily on the object itself (Figure 1). Once they have estimated COM for the boomerang, then you can check for students' understanding of COM by having them estimate COM on a kite, a toilet seat, and a tennis racket (Figure 5).

In the last Engage activity, students practice measuring COM of objects on a balance board. A simple, do-it-yourself balance board is constructed from plywood board (2 feet wide x 6 feet long, 3/4 inch thick) and two bathroom scales. The scales should be positioned mid-point at the long ends of the board and then zeroed to subtract the weight of the board 
from measurements (Figure 2). We suggest several balance boards per class so that students can work in small groups of three to six. Each group also needs a measuring tape.

Figure 5. Formative assessment of students' understanding of center of mass (COM). Using symmetry, geometry, and mass distribution, estimate COM for the objects below. Use lines to denote symmetry and geometry. Mark a circle to denote center of mass and write a justification statement to explain why you marked center of mass where you did.
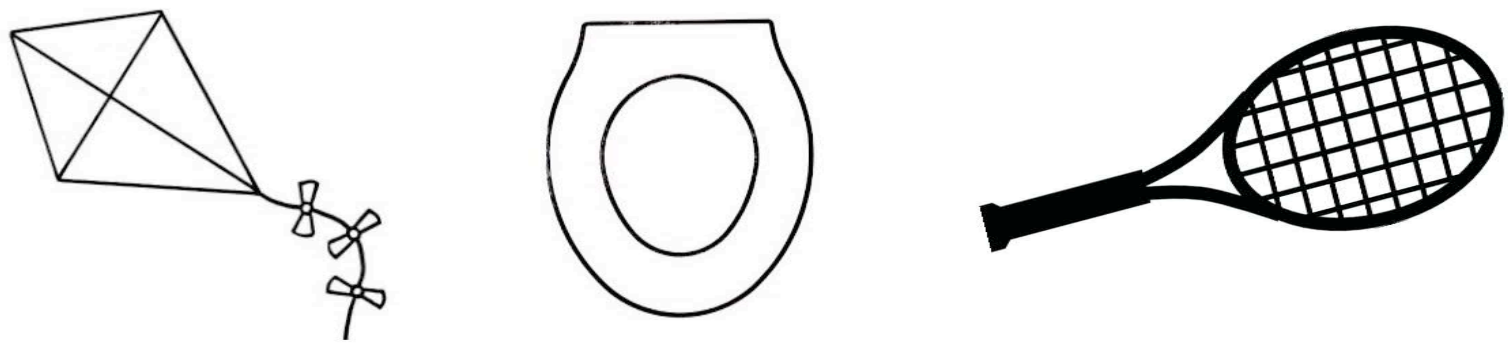

\begin{tabular}{|l|l|l|}
\hline Kite & Toilet seat & Tennis racket \\
\hline Justification: & Justification: & \\
& & \\
& & \\
\hline
\end{tabular}

Place a small stool or chair on the balance board and have a student sit on it. Identify the seated student's COM $(d)$ using the measurements from the two scales $\left(\mathrm{W}_{1}\right.$ and $\mathrm{W}_{2}$ ), the distance between the two scales (L) in the Balance Board Equation. Mark the COM location on the seated student with colored tape and repeat the measurement several times, removing and replacing the student and stool, to estimate measurement error associated with the balance board set-up (Figure 6). Safety note: Students should use caution getting on and off the balance board. There should be one student at each end to hold down the board as a student gets on and off the board. 
Figure 6. Student handout for estimating COM with the balance board during the Engage phase.

Use the balance board to measure the variables needed to calculate COM location $(d)$ of a teammate seated on a chair or stool. Conduct the test five times. Make sure to remove your teammate and stool completely from the board and place it at the same location each time. Note: $1 \mathrm{lb} .=4.45 \mathrm{~N}$

Distance between scales, L $(\mathrm{cm})$ :

\begin{tabular}{|c|c|c|c|}
\hline Trial & $\mathrm{W}_{1}(\mathrm{~N})$ & $\mathrm{W}_{2}(\mathrm{~N})$ & $d(\mathrm{~cm})$ \\
\hline 1 & & & \\
\hline 2 & & & \\
\hline 3 & & & \\
\hline 4 & & & \\
\hline 5 & & & \\
\hline
\end{tabular}

1) Calculate average COM location (d):

2) Identify minimum (d): and maximum (d):

3) Sketch the seated student on the stool and note COM on the sketch. Justify the COM location using symmetry, geometry, and mass distribution.

4) Explain why there is variability in your COM measurements and explain how you can minimize this variability.

\section{Explore (60 minutes)}

COM principles from Engage activities are applied to the case of a diver who is concerned about contacting the diving board during a flip dive. As the diver flips through the air, she spins about her COM with the outermost part of her body tracing a circle (Figure 7). The radius of this circle is called the flip radius ( $\mathrm{r}_{\text {flip }}$ ). If any part of this circle overlaps with the diving board, she is at risk of contacting the board. Therefore, she must jump away from the board at a distance equal to or greater than the flip radius. Flip radius will vary by body position during each dive. 
Students should develop a mathematical model to calculate flip radius based on measurements that can be obtained from a balance board experiment. To measure the flip radius, the diver would position herself on the balance board in the same body position as she uses during a flip dive. Her COM would be located using the Balance Board Equation, and the flip radius would be measured as the distance from the diver's COM to the outermost part of her body, most likely her feet, her head, or her hands depending on her diving position.

Figure 7. Illustration of flip radius. During a flip dive, the diver is translating and rotation about her COM, tracing a circle with her body as she does so. The diver must maintain clearance between the edge of the circle and the diving board. To clear the board, the diver must jump further than the flip radius ( $\mathrm{r}_{\text {flip }}$ ).

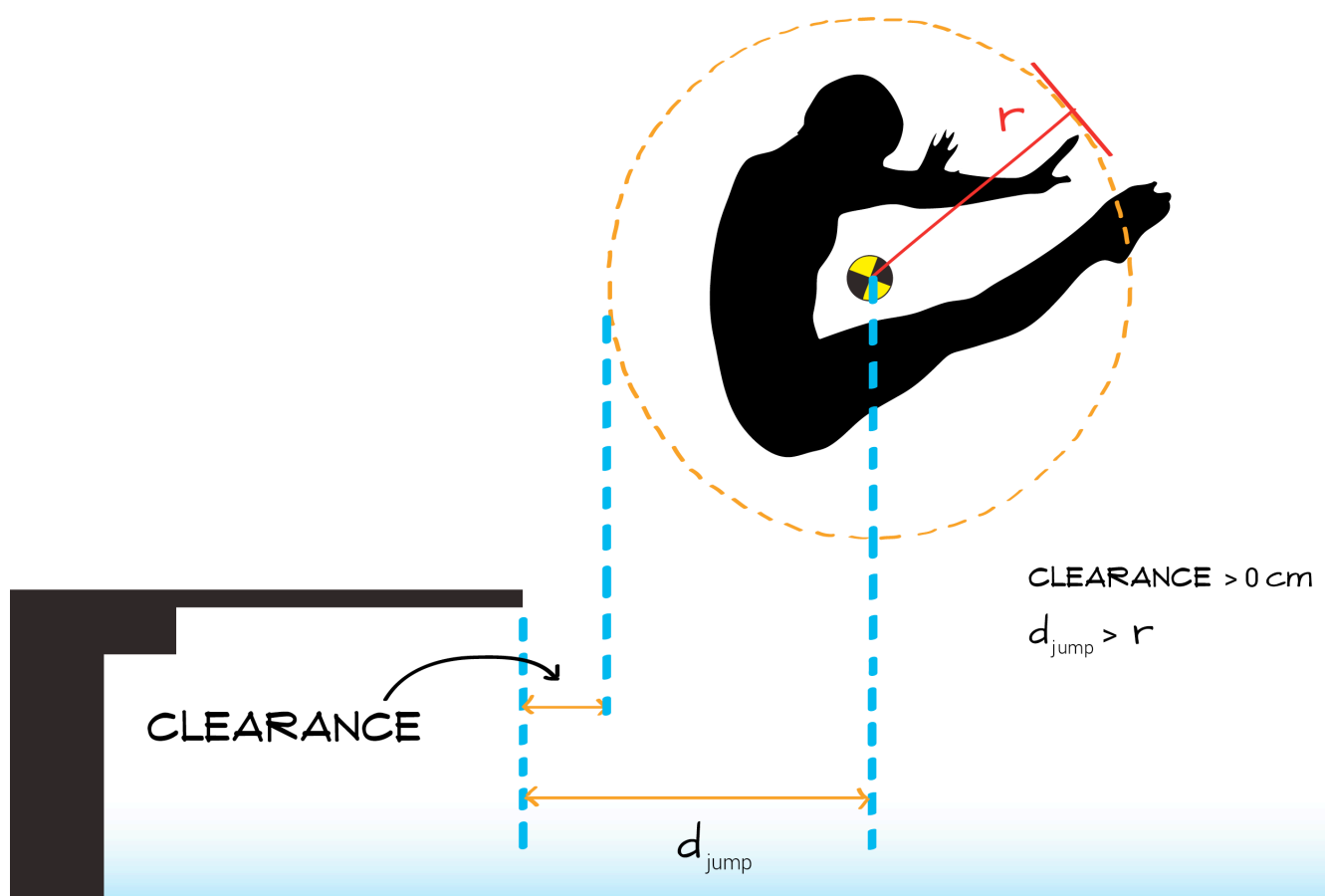


Students should hypothesize how flip radius for the diver may be affected by various factors, such as height, body shape, and body position during the flip. To test their hypotheses, students perform a balance board experiment with their group members as test subjects. Basic information should be collected on each test subject including sex and height (Figure 8) and a balance board experiment should be performed on each subject in multiple diving positions. Tables for data collection are shown in Figures 9 and 10.

Suggested positions include tuck, pike, straight with arms at side, and straight with arms overhead.

Figure 8. Setup and basic information for students to collect at the beginning of the Explore activity.

1) Set up the balance board. Zero both scales. Measure and record the distance between the two scales (L) in centimeters on the Data Collection Sheet.

2) Your test subjects are your group members. Randomly assign each person a 4-digit "Subject ID" so they are each to track on your Data Collection Sheet and also remain anonymous. Record the rest of the information on each subject in the table below.

\begin{tabular}{|l|l|l|l|l|}
\hline Subject ID & Name & Age (yrs) & Male/Female & Height (cm) \\
\hline & & & & \\
\hline & & & & \\
\hline & & & & \\
\hline & & & & \\
\hline & & & & \\
\hline
\end{tabular}

3) Develop hypotheses about how flip radius ( $\mathrm{r}_{\text {flip }}$ ) may vary by diving position. Do you expect flip radius to vary across subjects? Justify your hypotheses with an explanation.

Safety note: To avoid injury, test subjects should lay with their back on the board and then move their arms, legs, and head into the four diving positions. 
Students can combine group data into a whole class data set to draw more reliable conclusions about how flip radius, and hence, jumping distance may vary with the diver's height and body position during a dive. Students should also analyze potential relationships between flip radius and demographic data such as sex or height to discern correlated variables (Figures 9, 10, and 11).

Figure 9. Data collection sheet for the Explore phase activity.

\section{Data Collection Sheet}

Distance between scales, L $(\mathrm{cm})$ :

Note: 1 lb. $=4.45 \mathrm{~N}$

$\mathrm{p}=$ clearance distance $=$ edge of subject's body to edge of scale

$d=\frac{W_{2}}{W_{1}+W_{2}} L$

$\mathrm{r}_{\text {flip }}=d-\mathrm{p}$

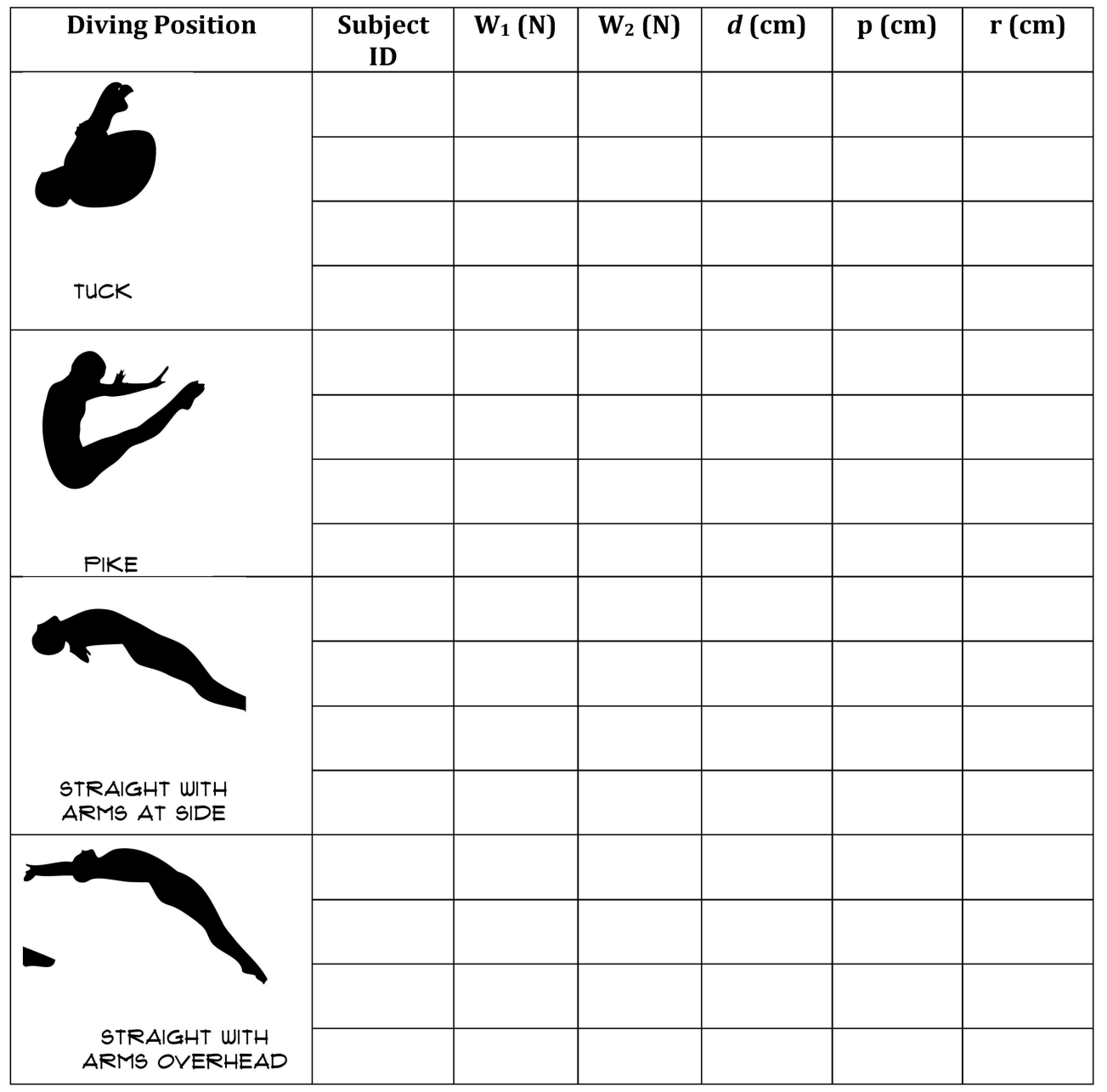


Figure 10. Illustration of the relationships among flip radius (r), jumping distance $(d)$ and clearance distance (p).

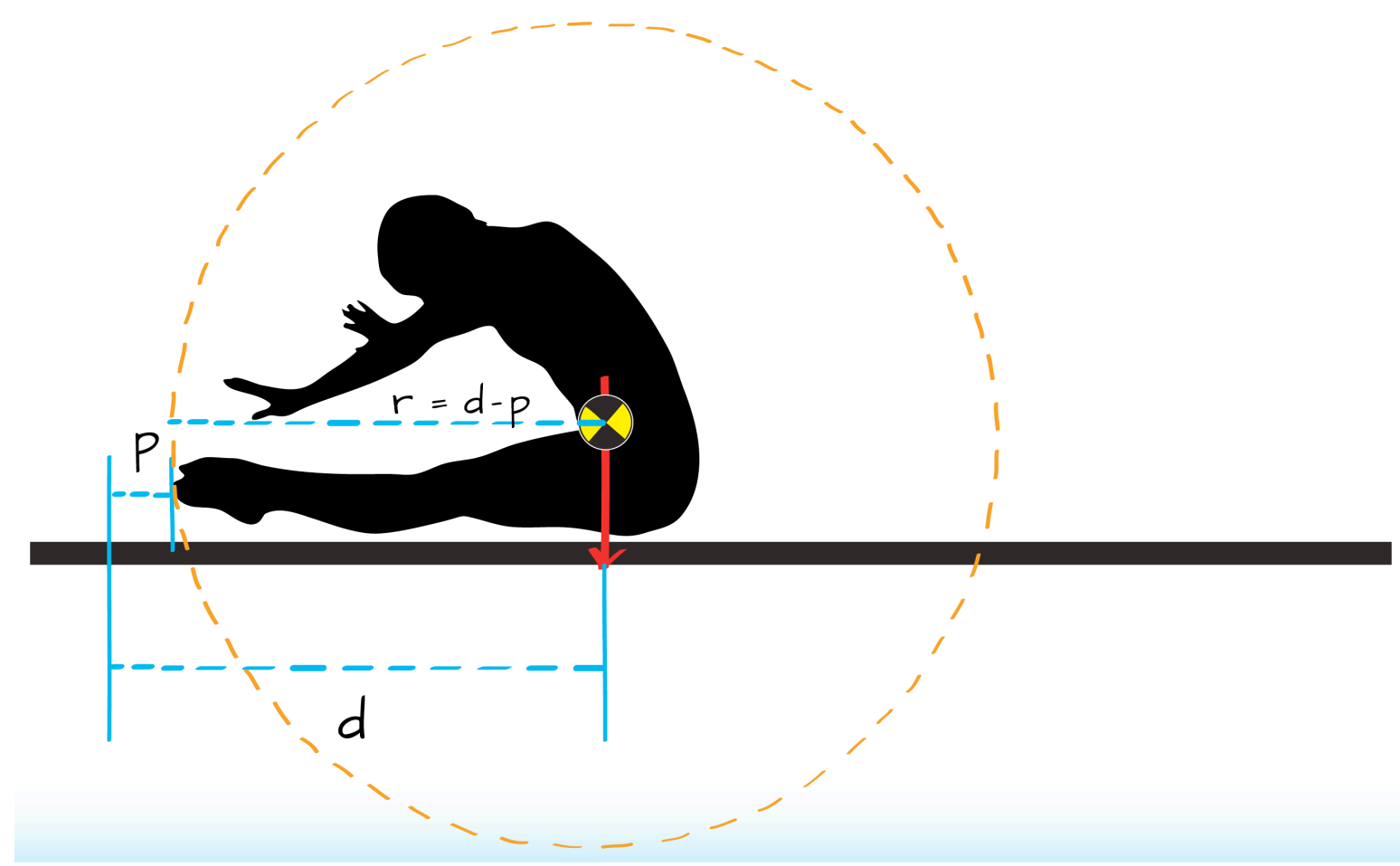


Figure 11. Data analysis prompts for Explore phase activity.

Use the data from your Data Collection Sheet to determine the average, minimum, and maximum flip radius across all subjects for each diving position. Create a graph to visually represent the data trends.

\begin{tabular}{|c|c|c|c|}
\hline \multirow{2}{*}{ Diving Position } & \multicolumn{3}{|c|}{ Flip radius, rflip (cm) } \\
\cline { 2 - 4 } Tuck & average & minimum & maximum \\
\hline Pike & & & \\
\hline $\begin{array}{c}\text { Straight, arms at } \\
\text { side }\end{array}$ & & & \\
\hline $\begin{array}{c}\text { Straight, arms } \\
\text { overhead }\end{array}$ & & & \\
\hline
\end{tabular}

Use the data from your Data Collection Sheet to determine the average, minimum, and maximum flip radius across all diving positions for each test subject. Create a graph to visually represent the data trends.

\begin{tabular}{|l|l|c|c|}
\hline \multirow{2}{*}{ Subject ID } & \multicolumn{3}{|c|}{ Flip radius, $\mathbf{r}_{\text {flip }}$ (cm) } \\
\cline { 2 - 4 } & average & minimum & maximum \\
\hline & & & \\
\hline & & & \\
\hline & & & \\
\hline & & & \\
\hline
\end{tabular}

Use the data from your Data Collection Sheet to determine the average, minimum, and maximum flip radius across all subjects for one or more demographic variable(s). For height or age, create sensible groups based on the data range. Create your own table below to organize the data and at least one graph to visually represent the data trends. 


\section{Explain (20 minutes)}

After examining data trends, students explain the relevance of their data by responding to writing prompts in Table 2. In the first and second prompts, students explain their data trends and whether the data supported or refuted their initial hypotheses. In the third prompt, students connect the data collected during the balance board experiment to the case of the diver who needs coaching on safe diving distances from the board.

Table 2. Writing prompts for students during the Explain phase.

Draw conclusions based on the data from your balance board experiment.

1) Describe the trends in your data. How flip radius vary with diving position? How does it vary across subjects? How does it vary based on demographic variables? Cite data from your tables and graphs to support your explanations.

2) Overall, did the balance board experiment support or refute your hypotheses? Explain why or why not, citing data from the experiment to support your statements.

3) Based on the data and analysis from your balance board experiment, make recommendations for a safe jumping distance for a diver for the four diving positions. In your recommendation, take into account the diver's sex, height, or age. Explain which variables you considered in making your recommendation, and support your recommendations with data from your experiment.

\section{Elaborate (time varies by activity)}

This lesson may be easily extended to involve hands-on biomechanics investigations. Students can perform similar balance board experiments for a range of athletic performance scenarios and/or to test specific hypotheses. For potential extension activities, see Table 3.

\section{Evaluate}

Formative and summative assessments are embedded into this $5 \mathrm{E}$ lesson.

Formative assessment includes checking students' ability to estimate COM using symmetry, geometry, and mass distribution (Figure 4) during the Engage phase. Once students have 
completed this formative assessment, it is worthwhile to engage students in while class discussion about the location of COM of the objects; this allows students to share their ideas and affords an opportunity for the teacher to redirect misconceptions or reinforce emerging ideas. The third writing prompt in the Explain phase serves as a summative assessment of students' understanding of COM; this prompt is designed to elicit students' ability to synthesize the outcomes of their balance board experiment with COM concepts.

Table 3. Possible lesson extensions to this lesson. All involve balance board experiments and can be performed by students on their classmates.

COM in the COM of the body in the "anatomically neutral" position (at rest with arms Population at side) is generally slightly above the navel for males and below for females

- Measure COM in body's anatomically neutral position, i.e., laying on back with arms resting at side. Normalize COM location to height of person and relate to anatomy, e.g., naval.

- Develop and test hypotheses about how COM location varies with body characteristics, e.g., height, build, and sex.

Squat Sitting or squatting is a common resting position for the body, and the form Form of the sit or squat varies across cultures.

- Measure COM location for shallow squat (knees slightly flexed), midrange squat (thighs parallel to floor), and deep squat (thighs touching calf).

- Compare COM location relative to anatomy in each case, specifically, where COM is located relative to the torso and to the feet.

- Assess comfort and perceived stability of each squat for the test subject. Develop hypotheses of how COM location affects comfort and stability in various squat positions.

Crutching Crutches are used to reduce weight bearing while walking - or ambulating - on an injured leg. Safe and effective ambulation keeps the body's COM near the mid-point of the body, i.e., in line with the naval.

- Measure COM location while standing with crutches. Experiment with one vs. two crutches and fully vs. partially offloading the "injured" leg.

- Develop and test hypotheses about which crutching configuration, e.g., one crutch with full offloading, is most and least balanced and efficient by comparing COM location while crutching to the body's mid-line. 


\section{References}

Bybee, R. (2009). The BSCS 5E instructional model and $21^{\text {st }}$ century skills. Washington, D.C.: National Academies Press.

National Governors Association Center for Best Practices (NGACBP). (2010). Common Core State Standards for mathematics and English language arts. Retrieved from: http://www.corestandards.org/

NGSS Lead States. (2013). Next generation science standards: For states, by states. Washington, D.C.: National Academies Press. 